\title{
BMJ Open Acupuncture for discomfort in patients during gastroscopy: a systematic review protocol
}

\author{
Weiming Wang, ${ }^{1,2}$ Tao Zhang, ${ }^{3}$ Weina Peng, ${ }^{1}$ Jiani Wu, ${ }^{1}$ Zhishun Liu ${ }^{1}$
}

To cite: Wang W, Zhang T, Peng W, et al. Acupuncture for discomfort in patients during gastroscopy: a systematic review protocol. BMJ Open 2014;4:e005735. doi:10.1136/bmjopen-2014005735

- Prepublication history for this paper is available online. To view these files please visit the journal online (http://dx.doi.org/10.1136/ bmjopen-2014-005735).

WW and TZ contributed equally.

Received 20 May 2014 Revised 17 August 2014 Accepted 18 August 2014

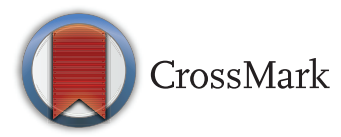

${ }^{1}$ Department of Acupuncture, Guang'anmen Hospital, China Academy of Chinese Medical Sciences, Beijing, China ${ }^{2}$ School of Graduates, Beijing University of Chinese Medicine, Beijing, China ${ }^{3}$ Department of Acupuncture, Beijing Hospital of Traditional Chinese Medicine, Capital Medical University, Beijing, China

Correspondence to Professor Zhishun Liu; liuzhishun@aliyun.com

\section{ABSTRACT \\ Introduction: This systematic review aims to assess the effectiveness and safety of acupuncture for discomfort in patients during gastroscopy.}

Methods and analysis: Randomised controlled trials will be searched electronically in several databases including OVID MEDLINE, EMBASE, the Cochrane Central Register of Controlled Trials (CENTRAL), Chinese Biomedical Literature Database (CBM), Chinese Medical Current Content (CMCC), Chinese Scientific Journal Database (VIP database), Wan-Fang Database and China National Knowledge Infrastructure (CNKI) from their respective founding dates to 30 April 2014. We will also try to find the literature by manually searching conference abstracts and reference lists. The study selection, extraction of data and assessment of study quality will be conducted independently by two researchers. Meta-analysis will be performed using RevMan V.5.2 statistical software. Data will be combined with either the fixed or random effect model based on a heterogeneity test. The results will be presented as a risk ratio for dichotomous data and standardised mean difference for continuous data.

Dissemination: This systematic review will evaluate the current evidence of acupuncture therapy for discomfort in patients during gastroscopy. The findings will be disseminated through peer-reviewed publication or conference presentations.

Trial registration number: PROSPERO CRD42014008966.

\section{INTRODUCTION}

Gastroscopy is an important method of gastrointestinal endoscopy in the diagnosis and treatment of digestive system diseases. It has been the most commonly performed endoscopic procedure, with an incidence of about 8.6/1000 of the population in the Trent region of the UK since the 1990s. ${ }^{1}{ }^{2}$ The number of patients receiving gastroscopy reached 0.2 million in Shanghai, China in $2001 .^{3}$ The average number of gastrointestinal endoscopes performed in 169 endoscopy units in China (all of the units possess gastroscopy) increased threefold in nearly

\section{Strengths and limitations of this study}

- To the best of our knowledge, there is only one systematic review related to acupuncture and gastrointestinal endoscopy, which was published in 2004 without any update until now. Our review will assess the effectiveness and safety of acupuncture for discomfort in patients during gastroscopy.

- The study selection, data extraction and quality assessment will be performed independently by two researchers. This will help ensure all relevant studies are included and not excluded for personal reasons.

- Japanese and Korean medical databases will not be included in our searches because of the language barrier. Hence, some relevant studies might be missed.

- The results of the systematic review may give gastroscopists more ways to help relieve patient discomfort during gastroscopy.

- Subgroup analysis will be used, as the reports to be reviewed use varying methods of acupuncture and varying measures of outcome, potentially making data analysis difficult.

12 years, from 2.3 /unit in 2001 to 9.3 /unit in 2013. It is believed that the number of patients receiving gastroscopy is continuously increasing because of the aging population, work pressure and dietary changes. ${ }^{4}$

During gastroscopy, gag reflex or distention of the gastric wall may be induced, which causes throat discomfort, nausea, retching or even emesis. ${ }^{5}{ }^{6}$ Moreover, gastroscopy can evoke anxiety, increase heart rate, lower blood oxygen and change blood pressure. ${ }^{7-9}$ Pharyngeal anaesthesia (eg, using lidocaine) and conscious sedation (eg, using diazepam, midazolam or propofol) are effective in minimising discomfort during gastroscopy. ${ }^{10-13}$ However, the use of these drugs increases the associated cost of the procedure and may cause retching during anaesthesia induction. ${ }^{5}$ Furthermore, potential risks of conscious sedation include respiratory and cardiovascular inhibition, 
hypotension or even coma. ${ }^{7} 14{ }^{15}$ For these reasons, unsedated gastrointestinal endoscopy (including gastroscopy) has been widely applied for many years and is still the major procedure selected by patients in China ${ }^{16}$ and other developing countries.

Acupuncture has a history of over 2000 years and plays an important role in complementary and alternative medicine. Recent study has suggested acupuncture as a way to increase tolerance and reduce discomfort during gastroscopy, ${ }^{17}$ with a number of clinical trials being conducted to assess the effectiveness of acupuncture therapy for discomfort during gastroscopy. ${ }^{18}{ }^{19}$ In the pre-retrieval of eight electronic databases, we have found more than 51 studies of acupuncture during gastroscopy.

There is so far only one published systematic review referring to 'acupuncture' and 'gastrointestinal endoscope'. ${ }^{20}$ The 2004 review assessed the effect of traditional manual acupuncture and electroacupuncture for gastrointestinal endoscopy. Six randomised controlled trials (RCTs) published from 1978 to 2003 were included and analysed in the review. However, no significant result was concluded as more high-quality RCTs with adequate sample size were needed. Although acupuncture originated from and is widely used in China, Chinese periodical databases were not searched in that review. In addition, three of the six included RCTs were acupuncture for colonoscopy. Thus, no definite conclusions on the effectiveness of acupuncture during gastroscopy could be drawn from that review. However, with a more superior search strategy and more included databases, we hope that our systematic review will obtain a more convincing conclusion.

Our systematic review aims to determine whether acupuncture is effective in reducing discomfort during gastroscopy and also whether acupuncture is safe for reducing discomfort during gastroscopy. In this article, we present the protocol of our proposed systematic review.

\section{METHODS AND ANALYSIS}

\section{Criteria for inclusion of studies in this review}

Types of studies

All clinical RCTs of acupuncture for discomfort in patients with gastroscopy will be included in the review, while randomised crossover studies and quasi-RCTs will be excluded. Because of the particularity of acupuncture manipulation, it is difficult to blind the acupuncturists. Therefore, blinding will not be part of the inclusion criteria, although it will be evaluated as an item in the risk of bias assessment.

\section{Types of participants}

Participants who underwent gastroscopy will be included, with no age limitation. Excluded participants will be those who have had a colonoscopy, and those who have suffered from chronic pharyngolaryngitis, severe digestive system diseases, persistent hiccups, severe nausea and retching, proven tumours in the upper digestive tract or uncontrolled cardiopulmonary disease.

\section{Types of interventions}

Any method of acupuncture usage will be included, such as manual acupuncture, electroacupuncture, fire needling, warm needling, pyonex, scalp acupuncture, auricular acupuncture and intradermal needling, without limitations on the treatment length and frequency. The control groups with no intervention, sham acupuncture, placebo control and drug therapy (such as lubricant use, pharyngeal anaesthesia and sedation) will be included. Acupuncture combined with another therapy will also be included if the combined therapy is the same in both groups. The combined interventions include drugs, but exclude complementary and alternative therapy such as relaxation and music therapy. To assess the efficacy of acupuncture therapy, we intend to compare acupuncture treatment with either no intervention or sham acupuncture. To assess the effectiveness of acupuncture therapies, we plan to compare them with conventional positive intervention consisting of lubricant use, pharyngeal anaesthesia and/or sedation.

\section{Types of outcome assessments}

The primary outcome measurement is the proportion of patients with discomfort as assessed via completed questionnaire or visual analogue scale (VAS). If the patient's condition permits, the proportion of throat discomfort, nausea, salivation, retching, emesis or hiccupping will be assessed. The secondary outcomes consist of the proportion of patients satisfied with the whole process and the proportion of patients who would opt for the same procedure again if required. If the included trials permit, the doses of anaesthesia or sedatives, the operation time of gastroscopy examination and the revival time of patients after gastroscopy will also be evaluated. The incidence of adverse events will be assessed as a safety outcome.

\section{Search methods for identification of studies Electronic searches}

We will electronically search the following databases from their founding date to 30 April 2014, regardless of the publication status: OVID MEDLINE, EMBASE, the Cochrane Central Register of Controlled Trials (CENTRAL), Chinese Biomedical Literature Database (CBM), Chinese Medical Current Content (CMCC), Chinese Scientific Journal Database (VIP database), Wan-Fang Database and China National Knowledge Infrastructure (CNKI). The search strategy has been decided on after a discussion among all reviewers according to the guidance of the Cochrane handbook..$^{21}$ The key words include "gastroscopy", "gastroscope", "endoscopy" or "endoscope" combined with "acupuncture", "manual acupuncture", "electroacupuncture", "fire needling", "warm needling", "pyonex", "scalp 
acupuncture", "auricular acupuncture" or "intradermal needling". The search strategy for OVID MEDLINE is shown in table 1. The search words used in Chinese databases have the same meaning as those used in the English databases.

\section{Other sources}

Potentially eligible studies will also be found through searching:

- The reference list of previously published reviews related to endoscopy and acupuncture;

- Conference abstracts that may have ongoing or unpublished trials in relation to gastroscopy and acupuncture. The data could be collected by contacting the author if applicable.

\section{Data collection and analysis}

Selection of studies

We plan to conduct this systematic review between 28 February 2014 and 30 November 2014. Prior to the

\begin{tabular}{|c|c|}
\hline Number & Search terms \\
\hline 1 & randomized controlled trial.pt. \\
\hline 2 & controlled clinical trial.pt. \\
\hline 3 & randomized.ab. \\
\hline 4 & randomised.ab. \\
\hline 5 & placebo.ab. \\
\hline 6 & randomly.ab. \\
\hline 7 & trial.ab. \\
\hline 8 & Groups.ab. \\
\hline 9 & or $1-8$ \\
\hline 10 & exp gastroscopy/ \\
\hline 11 & gastroscopy. ti, ab. \{Including Related Terms\} \\
\hline 12 & gastroscope. ti, ab. \{Including Related Terms\} \\
\hline 13 & endoscopy. ti, ab. \{Including Related Terms\} \\
\hline 14 & endoscope. ti, ab. \{Including Related Terms\} \\
\hline 15 & or $10-14$ \\
\hline 16 & exp acupuncture therapy, or acupuncture \\
\hline 17 & acupuncture. ti, ab. \{Including Related Terms\} \\
\hline 18 & $\begin{array}{l}\text { manual acupuncture. ti, ab. \{Including Related } \\
\text { Terms\} }\end{array}$ \\
\hline 19 & $\begin{array}{l}\text { electroacupuncture. ti, ab. \{Including Related } \\
\text { Terms }\}\end{array}$ \\
\hline 20 & fire needling. ti, ab. \{Including Related Terms\} \\
\hline 21 & warm needling, ti, ab. \{Including Related Terms $\}$ \\
\hline 22 & pyonex, ti, ab. \{Including Related Terms\} \\
\hline 23 & $\begin{array}{l}\text { scalp acupuncture, ti, ab. \{lncluding Related } \\
\text { Terms\} }\end{array}$ \\
\hline 24 & $\begin{array}{l}\text { auricular acupuncture, ti, ab. \{Including Related } \\
\text { Terms\} }\end{array}$ \\
\hline 25 & $\begin{array}{l}\text { intradermal needling, ti, ab. \{lncluding Related } \\
\text { Terms\} }\end{array}$ \\
\hline 26 & or $16-25$ \\
\hline 27 & 9 and 15 and 26 \\
\hline
\end{tabular}

This search strategy will be modified as required for other electronic databases. selection of the studies, all reviewers will undergo training to ensure a basic understanding of the background and purpose of the review. Reviewers will then reach a consensus on screening and later procedures. After electronic searching, the records will be moved to a database set up by EndNote software (V.X6). Records found through other sources will also be moved to the same database. Two reviewers (WW and TZ) will then independently determine the included studies using the following steps: first, remove duplicates (the same study published in different languages, or studies sharing the same title and abstract both in a journal and conference proceedings, or different articles reporting the same trial in different aspects); second, exclude studies in which participants accepted acupuncture treatment during colonoscopy, or studies in which participants received acupoint injections or other excluded interventions according to the exclusion criteria described above; third, remove studies that were not designed as randomised controlled trials, and trials in which no data can be extracted. The details of the study selection procedure are shown in a PRISMA flow chart (figure 1). During this procedure, any disagreement between the reviewers will be discussed and judged by a third author (ZL).

\section{Data extraction and management}

Before data extraction, all of the reviewers will jointly discuss and create a unified data extraction form. The following factors will be contained in the data extraction form:

1. General information including reference ID, author, time of publication, journal and location of performance.

2. Characteristics of participants.

3. Randomisation.

4. Method of allocation concealment.

5. Blinding.

6. Interventions, including the name of the intervention, length of treatment and type of control.

7. Outcome measures including primary and secondary outcomes.

8. Results.

9. Adverse events.

10. Conflicts of interest.

11. Other information such as the type of gastroscope.

The applicability of the form created will be tested by extracting information from three or more studies. After testing, WW and TZ will independently extract the data from the included studies and fill in the form. The final results of extraction will be checked, with any disagreement between the reviewers discussed and judged by a third author (ZL). ZL will also check the final data to make sure there are no errors.

\section{Assessment of risk of bias in included studies}

Two reviewers (WW and TZ) will evaluate the methodological quality of the included trials independently, with the use of the Cochrane Collaboration's tool for risk of 


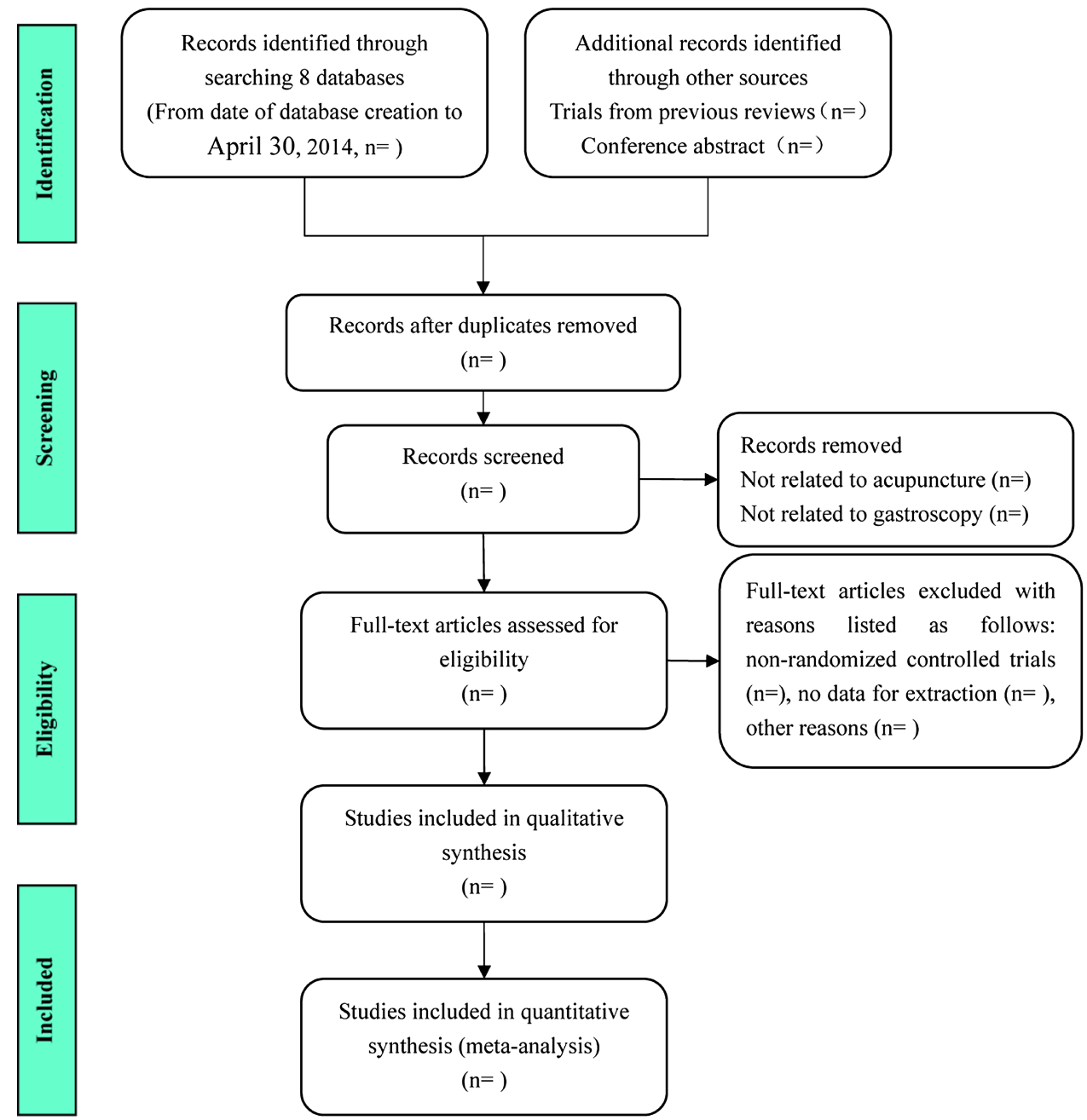

Figure 1 Flow diagram of the study selection process.

bias assessment. ${ }^{21}$ The tool consists of six domains of a trial, such as sequence generation, allocation concealment and blinding. The assessments will be categorised into three levels of bias: low risk, high risk and unclear risk.

\section{Measures of treatment effect}

For dichotomous outcomes, data will be analysed using a risk ratio (RR) with 95\% CIs. For continuous outcomes, a standard mean difference (SMD) with $95 \%$ CI will be used.

\section{Unit of analysis issues}

Because the gastroscopic procedure is a transient process and the acupuncture is often applied before and/or during the gastroscopy, the outcomes will usually be measured once the intervention is complete. Therefore, we will mainly focus on the instant effect of acupuncture therapy.

\section{Dealing with missing data}

If data required for the data extraction form are missing, we will try to contact the first or corresponding authors of the studies by telephone or email to obtain the information. If missing data are unobtainable, complete case analysis for continuous outcomes and dichotomous outcomes will be completed using the methods of Ebrahim et $a l^{22}$ and Akl et $a l,{ }^{23}$ with a sensitivity analysis then conducted.

\section{Assessment of heterogeneity}

We will perform the Higgins $\mathrm{I}^{2}$ test for heterogeneity prior to the meta-analysis to find out if inconsistencies exist within the included trials. We have set a cut-off point of $50 \%$ for the $\mathrm{I}^{2}$ statistics, as we consider that there will be significant heterogeneity among trials when an $\mathrm{I}^{2}$ value exceeds $50 \%$. If this is the case, meta-analysis will not be suggested.

\section{Assessment of reporting biases}

If 10 or more trials are included in a meta-analysis, we will generate funnel plots to assess the reporting biases. The plots will be assessed visually and by using Egger's test.

\section{Data synthesis}

Data synthesis will be performed using RevMan V.5.2 software from the Cochrane Collaboration. For 
dichotomous data, if no heterogeneity can be found, we will combine the RR of each study and compute $95 \%$ CI with the fixed effect model. If significant heterogeneity is detected, the random effect model will be used. For continuous data, we will combine the SMD of each study and calculate the $95 \%$ CI according to the outcome measurement.

\section{Subgroup analysis}

Subgroup analysis will be conducted according to different interventions, controls and outcome measures. If the included trials permit, we will also perform subgroup analysis based on the participants' ages and different diseases.

\section{Sensitivity analysis}

We will carry out a sensitivity analysis to remove the impact of lower quality studies if heterogeneity remains after subgroup analysis and input data verification. The meta-analysis will be repeated and studies of lower quality will be excluded. The result will be compared and discussed according to the pooled effect size.

\section{Ethics and dissemination}

This systematic review does not require formal ethical approval because the data we will use are not involved in individual data and privacy. The results of this review will provide a general overview and evidence of the effectiveness and safety of acupuncture therapy for discomfort in patients during gastroscopy. The findings will be disseminated through peer-reviewed publications or conference presentations.

\section{DISCUSSION}

The most common complications during gastroscopy are throat discomfort, nausea, retching and vomiting. ${ }^{5}$ The neural centre for vomiting is located at the reticular formation of the brain stem next to other vegetative nerve centres, such as the respiratory centre and vasomotor centre. The Chemoreceptor Trigger Zone (CTZ) exists at the area postrema of the vomiting centre, and transmits neurotransmitters to the centre. These neurotransmitters include dopamine, 5-hydroxytryptamine (5-HT) and substance $\mathrm{P}$. The vomiting centre is excited by the physical stimulation of the gastroscope, increase of neurotransmitters at CTZ and psychological factors. ${ }^{24}$ In addition, psychological factors such as anxiety or depression may raise the concentration of blood 5-HT and aggravate vomiting symptoms. Research indicates that acupuncture may adjust the function of vegetative nerves, regulate gastrointestinal tract function, protect gastric mucosa and have an antianxiety function that might reduce the concentration of blood $5-\mathrm{HT}^{25}$ Thus acupuncture may reduce the side effect of vomiting during gastroscopy. Similar mechanisms could be found for the other side effects. Acupuncture could have an effect on anatomical and physiopathological factors.
In this paper, we present a protocol for a systematic review of acupuncture for discomfort in patients during gastroscopy. With a history of over 2000 years, acupuncture therapy plays an important part in complementary and alternative medicine. Although clinical research has shown that acupuncture could reduce the discomfort generated by gastroscopy, to the best of our knowledge there is currently no high-quality review of these separate studies. Thus, this systematic review will analyse current clinical evidence on the effectiveness and safety of acupuncture for discomfort during gastroscopy. The results may benefit patients who undergo gastroscopy by minimising their associated discomfort.

As stated previously, the primary outcome of our systematic review is the removal of discomfort. The measurement of this outcome varies across different studies from a general description of the whole body to specific discomfort symptoms. Therefore, it may be difficult for the reviewers to pool the various data together, making subgroup analysis necessary. After a discussion among all the reviewers, we decided to divide subgroups according to the description of discomfort. Studies generally describing the discomfort of the whole body will be categorised and analysed as one group, while studies describing specific symptoms will be classified and analysed as another group. Moreover, Japanese and Korean medical databases will not be included in our searches because of the language barrier. Hence, some relevant studies might be missed.

Contributors WW and ZL contributed to the conception of the study. The manuscript of the protocol was drafted by WW and TZ, and was revised by JW and WP. The search strategy was developed by all authors and run by WW and TZ, who will also independently screen the potential studies, extract data of included studies, assess the risk of bias and finish data synthesis. ZL will arbitrate the disagreements and ensure that no errors occur during the study. All authors have approved the publication of the protocol.

\section{Competing interests None.}

Provenance and peer review Not commissioned; externally peer reviewed.

Data sharing statement Technical appendix, statistical code and dataset available from the corresponding author at Dryad repository, who will provide a permanent, citable and open access home for the dataset.

Open Access This is an Open Access article distributed in accordance with the Creative Commons Attribution Non Commercial (CC BY-NC 4.0) license, which permits others to distribute, remix, adapt, build upon this work noncommercially, and license their derivative works on different terms, provided the original work is properly cited and the use is non-commercial. See: http:// creativecommons.org/licenses/by-nc/4.0/

\section{REFERENCES}

1. Aljebreen AM. Unsedated endoscopy: is it feasible? Saudi $J$ Gastroenterol 2010;16:243-4.

2. Scott BB. Gastroenterology in the Trent region in 1992 and a review of changes since 1975. Gut 1995;36:468-72.

3. Xu GM, Fang YQ, Li ZS, et al. Survey of digestive endoscopy in Shanghai. Zhonghua Xiaohua Zazhi 2001;21:519-21.

4. Zhang XL, Lu ZS, Tang P, et al. Current application situation of gastrointestinal endoscopy in China. World $J$ Gastroenterol 2013;19:2950-5.

5. Keeffe EB. Towards safer endoscopy. In: Cotton PB, Tytgat GNJ Williams CBBowling TE, eds. Annual of gastrointestinal endoscopy. Rapid Science Publisher, 1997;1-14.

6. Borison HL, Borison R, McCarthy LE. Role of the area postrema in vomiting and related function. Fed Proc 1984;43:2955-8. 
7. Ozel AM, Oncü K, Yazgan Y, et al. Comparison of the effects of intravenous midazolam alone and in combination with meperidine on hemodynamic and respiratory responses and on patient compliance during upper gastrointestinal endoscopy: a randomized, double-blind trial. Turk J Gastroenterol 2008;19:8-13.

8. Hayward SR, Sugawa C, Wilson RF. Changes in oxygenation and pulse rate during endoscopy. Am Surg 1989;55:198-202.

9. Saijyo T, Nomura M, Nakaya $\mathrm{Y}$, et al. Assessment of autonomic nervous activity during gastrointestinal endoscopy: analysis of blood pressure variability by tonometry. $J$ Gastroenterol Hepatol 1998;13:816-20.

10. Campo R, Brullet E, Montserrat A, et al. Efficacy of low and standard midazolam doses for gastroscopy, a randomized, double-blind study. Eur J Gastroenterol Hepatol 2000;12:187-90.

11. Froehlich F, Schwizer W, Thorens J, et al. Conscious sedation for gastroscopy: patient tolerance and cardiorespiratory parameters. Gastroenterology 1995;108:697-704.

12. Trevisani L, Sartori S, Gaudenzi P, et al. Upper gastrointestinal endoscopy: are preparatory interventions or conscious sedation effective? A randomized trial. World J Gastroenterol 2004;10:3313-17.

13. Mulcahy HE, Greaves RR, Ballinger A, et al. A double-blind randomized trial of low-dose versus high-dose topical anaesthesia in unsedated upper gastrointestinal endoscopy. Aliment Pharmacol Ther 1996;10:975-9.

14. Iber FL, Sutberry M, Gupta R, et al. Evaluation of complications during and after conscious sedation for endoscopy using pulse oximetry. Gastrointest Endosc 1993;39:620-5.
15. Mokhashi MS, Hawes RH. Struggling toward easier endoscopy. Gastrointest Endosc 1998;48:432-40.

16. Wang HL, Ye F, Liao WF, et al. Unsedated versus sedated gastrointestinal endoscopy: a questionnaire investigation in Wuhan, central China. J Huazhong Univ Sci Technolog Med Sci 2013;33:857-61.

17. Lazzaroni M, Bianchi Porro G. Preparation, premedication, and surveillance. Endoscopy 2005;37:101-9.

18. Cahn AM, Carayon P, Hill C, et al. Acupuncture in gastroscopy. Lancet 1978;311:182-3.

19. Tarçin O, Gürbüz AK, Poçan $S$, et al. Acustimulation of the neiguan point during gastroscopy: its effects on nausea and retching. Turk $J$ Gastroenterol 2004;15:258-62.

20. Lee H, Ernst E. Acupuncture for Gl endoscopy: a systematic review. Gastrointest Endosc 2004;60:784-9.

21. Higgins JPT, Green S. Cochrane handbook for systematic reviews of interventions version 5.1. O [updated March 2011]. The Cochrane Collaboration, 2011

22. Ebrahim S, Akl EA, Mustafa RA, et al. Addressing continuous data for participants excluded from trial analysis: a guide for systematic reviewers. J Clin Epidemiol 2013;66:1014-21.

23. Akl EA, Johnston BC, Alonso-Coello $\mathrm{P}$, et al. Addressing dichotomous data for participants excluded from trial analysis: a guide for systematic reviewers. PLOS ONE 2013;8:e57132.

24. Grunberg SM, Hesketh PJ. Control of chemotherapy-induced emesis. N Engl J Med 1993;329:1790-6.

25. Wang SM, Peloquin C, Kain ZN. The use of auricular acupuncture to reduce preoperative anxiety. Anesth Analg 2001;93:1178-80. 